\title{
Management des Praxiserfolges
}

Am 6. und 7. Mai 2016 lädt der Freie Verband Deutscher Zahnärzte (FVDZ) zum 11. Praxis-Ökonomie-Kongress nach Westerland auf Sylt ein. Auf die Kongressteilnehmer wartet ein vielseitiges Programm, das gezielt auf die Bedürfnissen von Praxisinhabern ausgerichtet ist. Experten beleuchten in Vorträgen betriebswirtschaftliche und ökonomische Themen aus unterschiedlichen Blickwickeln. Dazu zählen unter anderem das Management komplexer Praxisstrukturen, die wichtigsten Punkte bei einer Praxiskooperation oder Faktoren für eine erfolgreiche Mitarbeiter-Motivation. Es gibt noch wenige Plätze beim Praxis-Ökonomie-Kongress auf der größten ostfriesischen Insel.

Weitere Infos finden Sie im Internet unter www.fvdz.de. Anmeldungen sind auch direkt möglich mit dem Service-Coupon auf Seite 105.

Hier steht eine Anzeige. Springer

\section{Jetzt noch buchen}

\section{Sommerkongress des Freien Verbands auf Usedom}

Auch wenn der Frühling erst langsam in Fahrt kommt, wirft ein Termin Ende nächsten Monats schon jetzt große Schatten voraus: Vom 23. bis 27. Mai steht auf der sonnenverwöhnten Insel Usedom der 23. Sommerkongress des Freien Verbands Deutscher Zahnärzte (FVDZ) auf dem Programm.

Zahnärzte und Praxismitarbeiter erwartet in Heringsdorf neben Sonne, Sand und Meer wieder eine abwechslungsreiche und niveauvolle Fortbildung. Nähere Informationen sowie das komplette Kongress-Programm gibt es in der FVDZ-Bundesgeschäftsstelle unter Tel. 0228-8557-0 oder im Internet unter www. fvdz.de.
Hier steht eine Anzeige.

Springer

\section{Europa und Praxistätigkeit im Fokus beim Berliner Wochenende}

Ganz im Zeichen von Europa und einer erfolgreichen Praxistätigkeit steht das diesjährige Fortbildungswochenende, zu dem der Freie Verband Deutscher Zahnärzte (FVDZ) als Teil des Mentoringprogramms am ersten Juniwochenende nach Berlin einlädt. An zwei Tagen wartet auf Zahnärzte, Assistenten und Studierende ein abwechslungsreiches Fort-

Hier steht eine Anzeige.

Springer
Freiberuflichkeit. Was bedeuten die Entscheidungen aus Brüssel für den Praxisalltag der Zahnärzteschaft? Wie wird die EUPolitik künftig Einfluss auf die freie Berufsausübung nehmen? Auf diese und viele weitere Fragen wird die gesundheitspolitische Expertin vom Rednerteam der EU-Kommission, Ulla Kalbfleisch-Kottsieper, in einem Vortrag eingehen. Unter dem Motto „Sicher in die Zukunft" geht es in den Vorträgen am Samstag, 4. Juni, vor allem um Abrechnungsfragen.

Die Kosten für An- und Abreise, Verpflegung und Übernachtung sind selbst zu tragen, die Teilnahme an den Vorträgen ist kostenfrei. Wegen der begrenzten Teilnehmerzahl wird um eine Anmeldung für beide Tage oder für einzelne Programmpunkte gebeten. Das detaillierte Programm steht auf der Verbandswebsite unter der Rubrik „FVDZ praxis“. Dort ist auch ein Formular zur direkten Anmeldung. Weitere Informationen gibt es bei der Bundesgeschäftsstelle (Ansprechpartnerin: Frauke Garstka, fg@fvdz.de, Tel. 0228 8557-32). 